\title{
Quantum phase transition of nonlinear light in the finite size Dicke Hamiltonian
}

\author{
B. M. Rodríguez-Lara* and Ray-Kuang Lee \\ Institute of Photonic Technologies, National Tsing-Hua University, Hsinchu 300, Taiwan \\ *Corresponding author: bmlara@mx.nthu.edu.tw
}

Received August 10, 2010; revised September 14, 2010; accepted September 17, 2010; posted September 21, 2010 (Doc. ID 133215); published October 29, 2010

\begin{abstract}
We study the quantum phase transition of an $\mathrm{N}$ two-level system ensemble interacting with an optical degenerate parametric process, which can be described by the finite size Dicke Hamiltonian plus counter-rotating and quadratic field terms. Analytical closed forms of the critical coupling value and their corresponding separable ground states are derived in the weak and strong coupling regimes. The existence of bipartite entanglement between the two-level system ensemble and photon field as well as between ensemble components for moderate coupling is shown through numerical analysis. Given a finite size, our results also indicate the coexistence of squeezed fields and squeezed atomic ensembles. (c) 2010 Optical Society of America

OCIS codes: $270.0270,270.5580,270.5585$.
\end{abstract}

\section{INTRODUCTION}

The study of light-matter interaction has been the central topic of quantum optics; it has laid the foundation for laser theory, quantum state engineering, fundamental testing of quantum mechanics, and implementation of quantum information processing [1]. Among the various systems involving the interaction of photons and atoms, the simplest and the most important building block to illustrate interesting quantum phenomena involves just the one two-level atom (TLA) [2]. As the number of TLAs increase, collective effects give rise to intriguing manybody phenomena; e.g. the existence of a coherent superradiant phase at zero temperature [3].

The Hamiltonian describing the coupling of a noninteracting atomic ensemble with a single quantized electromagnetic field mode is equivalent, via a Power-Zineau transformation, to the Hamiltonian of a free particle under a field related potential [1]. The ground state energy of such a system is bounded from below by the atomic ground state and the vacuum field, ergo a super-radiant phase transition of the ground state in a charge only system coupled to a single field mode is not possible $[4,5]$. Within the standard minimal coupling, long wavelength, and rotating wave approximation-and discarding quadratic terms - the interaction of a photon field with an ensemble of TLAs is described by the Dicke Hamiltonian,

$$
\hat{H}_{\text {Dicke }}=\hbar \omega_{p} \hat{a}^{\dagger} \hat{a}+\hbar \omega_{a} \hat{J}_{z}+\hbar \frac{\lambda}{\sqrt{N}}\left(\hat{a} \hat{J}_{+}+\hat{a}^{\dagger} \hat{J}_{-}\right)
$$

where the transition energy for each one of the $N$ TLAs and the radiation field frequency are $\omega_{a}$ and $\omega_{p}$, in that order. The atomic ensemble operators are defined in terms of the Pauli matrices for the $j$ th TLA as $\hat{J}_{z}$ $=(1 / 2) \sum_{j=1}^{N} \sigma_{z}^{(j)}$ and $\hat{J}_{ \pm}=\sum_{j=1}^{N} \sigma_{ \pm}^{(j)}$. The coupling strength for the photon-atom interaction is denoted by $\lambda$. The impossibility for a quantum phase transition (QPT) in the source model translates into a violation of the ThomasReiche-Kuhn (TRK) sum rule for an atom, if the relation between coupling strength and quadratic parameter for a phase transition in the Dicke model were to occur [6]. In order to observe the effect of the radiation-matter coupling known as super-radiant phase transition in charge only systems, more realistic models, such as spin magnetic moment, statistics, or infinitely many electromagnetic field modes, should be taken into account [7].

Despite the fact that a QPT is forbidden in the physical system that originated the Dicke model, the phenomenon is interesting by itself. First, the existence of a QPT in the Dicke Hamiltonian was reported as a series of instabilities of the ground state for a finite size ensemble of twolevel systems [8]. Then, the existence of a super-radiant thermodynamic phase transition was proved for an infinitely large ensemble interacting with a coherent boson field at a given temperature [9-11]. Also, in the classical limit, studies indicated that this quantum critical phenomenon is associated with quantum chaos and ensemble-field entanglement [12-14]. Non-trivial scaling exponents at the critical point have been discussed on the large ensemble size regime [15]. Bipartite intra-ensemble entanglement resulting from finite size effects, was demonstrated for the Dicke Hamiltonian [16]. This theoretical understanding has motivated proposals for the realization of the Dicke model in systems that might allow a super-radiant phase transition; e.g., open dynamical systems involving semiconductor quantum wells or quantum dots $[17,18]$, open dynamical cavity-QED systems with neutral atoms [19] and ions [20], and superconducting quantum devices $[21,22]$, just to mention a few. Recently, it has been shown that a standing-wave laser driven Bose-Einstein condensate (BEC) coupled to a high finesse optical cavity accounting for the center of mass motion realizes the Dicke model, and that the super-radiant phase corresponds to a periodical self-organized phase of the atoms $[23,24]$. 
Currently, there is great interest in pursuing quantum phase transitions of light, since photons interacting with atoms should be much easier to study and probe than electrons in condensed matter systems [25,26]. Schemes to realize composite Dicke models have been proposed, e.g., by combining photon hopping between identical cavities in the photon-blockade regime. Mott-insulator to superfluid QPT has been demonstrated in the Dicke-BoseHubbard model for an arbitrary number of two-level atoms [27]. More exotic QPTs of light have been predicted in a Heisenberg spin 1/2 Hamiltonian [28], two species of Bose-Hubbard model [29], arrays of coupled cavities [30,31], and dual-species optical-lattice cavities [32]. These studies have brought the possibility of analyzing critical quantum phenomena in conventional condensed matter systems by manipulating the interaction between photons and atoms.

As nonlinear optics plays an important role in quantum optics, especially in the generation of quantum noise squeezed states [33,34], a natural question one may ask is how to associate nonlinear quantum processes with the phenomenon of QPT. Sub-Poissonian photon statistics of the field state and momentum squeezing of the atomic state have been predicted for the Dicke model [14]. It also has been proposed that squeezing of the photon field carries signatures of the associated quantum critical phenomena in the size-consistent Dicke model [35].

In this work, we study the quantum critical phenomena of $N$ two-level systems embedded within a nonlinear optical medium, with $N$ finite. An optical degenerate parametric down conversion (PDC) process, where the nonlinear medium is pumped by a classical field of frequency $2 \omega_{f}$ and that field is converted into pairs of identical photons of frequency $\omega_{f}$ each, is considered. The corresponding nonlinear interaction Hamiltonian is given by

$$
\hat{H}_{\mathrm{PDC}}=\hbar \kappa\left(\hat{a}^{2}+\hat{a}^{\dagger 2}\right),
$$

where the nonlinear parameter $\kappa=\chi^{(2)} \beta$ is defined by the second-order nonlinearity coefficient $\chi^{(2)}$ and the classical amplitude of the pumping field $\beta$. By plugging the degenerate PDC Hamiltonian, Eq. (2), into the Dicke model, Eq. (1), and restoring the counter-rotating terms we obtain the following Hamiltonian:

$$
\hat{H}=\hbar \omega_{f} \hat{a}^{\dagger} \hat{a}+\hbar \omega_{a} \hat{J}_{z}+\hbar \frac{\lambda}{\sqrt{N}}\left(\hat{a}+\hat{a}^{\dagger}\right) \hat{J}_{x}+\hbar \kappa\left(\hat{a}+\hat{a}^{\dagger}\right)^{2},
$$

where we have rescaled the system energy by $(-\hbar \kappa)$ and redefined the photon frequency as $\omega_{f}=\omega_{p}-2 \kappa$. In the literature, the Hamiltonian in Eq. (3) is that of a noninteracting TLAs ensemble driven by an electromagnetic field with the standard minimal coupling in the long wavelength limit. Of course, such a QPT does not exist for charge-only systems interacting with a finite number of electromagnetic radiation modes [4-7], but the proposed nonlinear optics PDC process may provide a strong nonlinearity for the field, which might be coupled to a feasible realization of the Dicke model [17-24,36-38]. The theoretical quantum phase transition of the proposed model, Eq. (3), has been shown in the thermodynamic limit, i.e., both ensemble size and volume are considered infinitely large, and $N$ and $V \rightarrow \infty[39]$. To the knowledge of the au- thors, the finite size effect on the QPT of this Hamiltonian remains unanswered.

The purpose of this work is twofold. First, we show analytically that a pair of unitary transformations effect a rotating wave approximation equivalence on the Hamiltonian in the weak coupling regime; thus the exact critical coupling strength can be calculated [16]. In the strong coupling regime, we show how the critical coupling strength can be calculated under just a semi-classical field approximation. In both cases, the critical coupling value of the finite-size $N$ Dicke Hamiltonian plus counterrotating and quadratic field terms is independent of the atomic number and agrees with results derived from the classical limit, $N$ and $V \rightarrow \infty$. Second, treated as a degenerate parametric process, we numerically demonstrate the existence of squeezed fields and squeezed atomic ensembles as well as bipartite entanglement between the two-level system ensemble and photon field and among ensemble components themselves.

\section{WEAK COUPLING REGIME, $\lambda \ll \omega_{a}$}

In order to derive a set of critical coupling values for the quantum phase transition, we first consider the weak coupling regime, i.e., $\lambda \ll \omega_{a}$. In analogy to the unitary squeezed operator for a degenerate PDC, we define the following unitary transformation and associated parameter:

$$
\hat{T}=e^{\eta\left(\hat{a}^{2}-\hat{a}^{\dagger 2}\right)}, \quad \eta=\kappa /\left[2\left(\omega_{f}+2 \kappa\right)\right] .
$$

Under the restriction $\eta \ll 1$, the Hamiltonian in Eq. (3) is reduced to

$$
\begin{gathered}
\tilde{H}=\hat{T}^{-1} \hat{H} \hat{T}, \\
\approx \hbar \widetilde{\omega}_{f} \hat{a}^{\dagger} \hat{a}+\hbar \frac{\omega_{a}}{2} \hat{J}_{z}+\hbar \frac{\widetilde{g}}{\sqrt{N}}\left(\hat{a}+\hat{a}^{\dagger}\right) \hat{J}_{x}+\hbar \widetilde{\kappa} .
\end{gathered}
$$

Thus, the original Hamiltonian, Eq. (3), is approximated by the well-known Dicke Hamiltonian plus the counter rotating terms with a modified field frequency $\widetilde{\omega}_{f}$, a modified coupling constant $\widetilde{g}$, and a constant energy shift $\hbar \widetilde{\kappa}$, defined as

$$
\tilde{\omega}_{f}=\left(\frac{\omega_{f}+4 \kappa}{\omega_{f}+2 \kappa}\right) \omega_{f}, \quad \widetilde{g}=\left(\frac{\omega_{f}+\kappa}{\omega_{f}+2 \kappa}\right) \lambda, \quad \widetilde{\kappa}=\left(\frac{\omega_{f}}{\omega_{f}+2 \kappa}\right) \kappa
$$

As the total excitation number, $\hat{N}=\hat{a}^{\dagger} \hat{a}+\hat{J}_{z}$, does not commute with this Hamiltonian, Eq. (5), in order to further simplify our problem, a second unitary transformation is used:

$$
\hat{U}=e^{-l \xi\left(\hat{a}+\hat{a}^{\dagger}\right) \hat{J}_{y}}, \quad \xi=\widetilde{g} /\left[\sqrt{N}\left(\omega_{a}+\widetilde{\omega}_{f}\right)\right],
$$

where the newly defined parameter fulfills $\xi \ll 1$ due to the weak coupling regime requirement $\lambda \ll \omega_{a}$. Neglecting all but linear powers of the parameter $\xi$, the Hamiltonian in Eq. (3) is written

$$
\hat{H}_{W}=\hat{U}^{-1} \hat{T}^{-1} \hat{H} \hat{T} \hat{U}
$$




$$
\approx \hbar \widetilde{\omega}_{f} \hat{a}^{\dagger} \hat{a}+\hbar\left[\omega_{a}+\widetilde{\omega}_{a}\left(\hat{a}+\hat{a}^{\dagger}\right)^{2}\right] \hat{J}_{z}+\hbar \frac{\tilde{\lambda}}{\sqrt{N}}\left(\hat{\hat{a}} \hat{J}_{+}+\hat{a}^{\dagger} \hat{J}_{-}\right)
$$

with the extra frequency $\widetilde{\omega_{a}}$ and the modified coupling $\tilde{\lambda}$ given by the expressions

$$
\widetilde{\omega}_{a}=\frac{2 \widetilde{g}^{2}}{N\left(\omega_{a}+\widetilde{\omega}_{f}\right)}, \quad \tilde{\lambda}=\frac{2 \widetilde{\omega}_{f} \widetilde{g}}{\left(\omega_{a}+\widetilde{\omega}_{f}\right)} .
$$

The weak regime assumption makes it possible to neglect the extra frequency $\widetilde{\omega}_{a}$. Thus the weak limit Hamiltonian, Eq. (8), is further reduced to the well-known finite size Dicke model in Eq. (1), of which the ground state can be found exactly and undergoes a phase transition at the critical value $\widetilde{\lambda}=\sqrt{\omega_{a} \widetilde{\omega}_{f}}[16,40]$. In our case, the critical coupling value in the weak coupling regime can be explicitly expressed as

$$
\lambda_{W} \approx \frac{\omega_{f}\left(\omega_{a}+\omega_{f}\right)+2 \kappa\left(\omega_{a}+2 \omega_{f}\right)}{2\left(\omega_{f}+\kappa\right)} \sqrt{\frac{\omega_{a}\left(\omega_{f}+2 \kappa\right)}{\omega_{f}\left(\omega_{f}+4 \kappa\right)}} .
$$

For coupling values fulfilling the condition $\lambda \ll \lambda_{W}$, we can write the ground state and the corresponding energy of our system as

$$
\begin{aligned}
& \left|G_{W}\right\rangle=\hat{T}|0\rangle \underset{j=1}{\otimes}|g\rangle_{j} \approx \frac{|0\rangle-\eta|2\rangle}{\sqrt{\eta^{2}+1}} \underset{j=1}{\otimes}|g\rangle_{j}, \\
& E_{G_{W}}=\hbar\left(\widetilde{\kappa}-\frac{N \omega_{a}}{2}\right),
\end{aligned}
$$

where $|g\rangle_{j}$ denotes the ground state for the $j$ th TLAs. The ground state is a pure separable state and independent of the size of the atomic ensemble. Unlike the Dicke model, here the field is in a superposition of vacuum $|0\rangle$ and twophoton $|2\rangle$ states as a result of the degenerate parametric process.

\section{STRONG COUPLING REGIME, $\lambda \gg \omega_{a}$}

In the weak coupling regime, the ground state is well described by a finite superposition of Fock states times the two-level system ground state, while in the strong coupling regime, it is possible to consider the field in a coherent state and try to find the corresponding ensemble state. By substituting the photon creation and annihilation operators by their expectation values, the Hamiltonian in Eq. (3) becomes

$$
\hat{H}_{S}=\hbar \omega_{f}|\alpha|^{2}+\hbar \kappa \alpha_{R}^{2}+\hbar \omega_{a} \hat{J}_{z}+\frac{2 \hbar \lambda}{\sqrt{N}} \alpha_{R} \hat{J}_{x}
$$

where the complex coherent state parameter is defined as $\alpha=\alpha_{R}+\imath \alpha_{I}$. It is possible to arrange this semi-classical Hamiltonian in Eq. (12) as a nested array of tensor products of the form

$$
\begin{aligned}
\hat{H}_{S}= & \hbar\left(\omega_{f}|\alpha|^{2} \kappa \alpha_{R}^{2}\right) \mathbb{I}_{2^{N}}+\left\{\left[(\ldots) \otimes \mathbb{I}_{2}+\mathbb{I}_{2^{N-2}} \otimes \hat{H}_{2}\right] \otimes \mathbb{I}_{2}+I_{2^{N-1}}\right. \\
& \left.\otimes \hat{H}_{2}\right\}
\end{aligned}
$$

where the symbol $I_{d}$ represents the unit matrix of dimension $d$, and the auxiliary matrix of dimension two is

$$
\hat{H}_{2}=\hbar\left(\frac{\omega_{a}}{2} \hat{\sigma}_{z}+\frac{2 \lambda \alpha_{R}}{\sqrt{N}} \hat{\sigma}_{x}\right) .
$$

Thus, the ground state energy is found as

$$
E_{G_{S}}=\hbar\left[\omega_{f}|\alpha|^{2}+\kappa \alpha_{R}^{2}-\frac{N}{2} \sqrt{\omega_{a}^{2}+16 \lambda^{2} \alpha_{R}^{2} / N}\right] .
$$

In order to calculate the critical coupling value, we optimize this ground state energy for the real and imaginary parts of the coherent state parameter, $\alpha$, and find the following self-consistency equations:

$$
\begin{aligned}
\alpha_{R}^{2} & =\frac{N\left[16 \lambda^{4}-\omega_{a}^{2}\left(\omega_{f}+4 \kappa\right)^{2}\right]}{4 \lambda^{2}\left(\omega_{f}+4 \kappa\right)^{2}}, \\
\alpha_{I} & =0 .
\end{aligned}
$$

The phase transition in the strong coupling regime occurs at the critical value given by the expression

$$
\lambda_{S}=\frac{1}{2} \sqrt{\omega_{a}\left(\omega_{f}+4 \kappa\right)} .
$$

Although a finite size has been assumed for the atomic ensemble, this critical coupling value found in the strong coupling regime, Eq. (17), is in accord with that derived from the free energy by using the thermodynamic limit method for an infinitely large two-level system ensemble $[6,10,11]$ for the reason that in both cases the field is assumed to be in a coherent state.

The mean-field constrain set, Eq. (16), approximates, in the strong coupling regime $\lambda \gg \lambda_{S}$, the following ground state and ground state energy:

$$
\begin{aligned}
& \left|G_{S}\right\rangle=|\alpha\rangle \underset{j=1}{N}|v\rangle_{j}, \\
& E_{G_{S}}=-\frac{\hbar N\left[16 \lambda^{4}+\omega_{a}^{2}\left(\omega_{f}+4 \kappa\right)^{2}\right]}{16 \lambda^{2}\left(\omega_{f}+4 \kappa\right)},
\end{aligned}
$$

where the auxiliary two-level state is defined as

$$
\begin{gathered}
|v\rangle=\frac{1}{\sqrt{\beta^{2}+1}}(|g\rangle+\beta|e\rangle), \\
\beta=\frac{\omega_{a}\left(\omega_{f}+4 \kappa\right)-4 \lambda^{2}}{\sqrt{16 \lambda^{4}-\omega_{a}^{2}\left(\omega_{f}+4 \kappa\right)^{2}}} .
\end{gathered}
$$

Again, as expected, the ground state is a pure separable state; here the difference is that each component of the ensemble is in a superposition of the ground, $|g\rangle$, and excited states, $|e\rangle$. Furthermore, for a coupling parameter larger than the nonlinear parameter, $\lambda \gg \kappa$, the auxiliary state is the balanced superposition $|v\rangle=(|g\rangle-|e\rangle) / \sqrt{2}$ with null population difference, $\left\langle\hat{\sigma}_{z}\right\rangle=0$. 


\section{MODERATE COUPLING REGIME}

Besides the weak and strong coupling regimes, where the ground states are both separable states, we apply a direct numerical calculation to find the ground state in the moderate coupling regime. In the simulation, each and every single two-level system is taken to be indistinguishable from each other, and the angular momentum eigenstates basis is used:

$$
\begin{aligned}
& \hat{J}_{z}|N / 2, m\rangle=m|N / 2, m\rangle, \\
& \hat{J}_{ \pm}|N / 2, m\rangle=\sqrt{\frac{N}{2}\left(\frac{N}{2}+1\right)-m(m \pm 1)}|N / 2, m \pm 1\rangle,
\end{aligned}
$$

where the Dicke state $|N / 2, m\rangle$ is the superposition of all possible ensemble states with $N / 2+m$ two-level systems in the excited state and the rest, $N / 2-m$, in the ground state, such that $m=-N / 2,-N / 2+1, \ldots, N / 2-1, N / 2$. In the Fock and Dicke basis, for the field and the ensemble in that order, the matrix for the Hamiltonian is given by-in units of $\hbar-$

$$
\begin{aligned}
\hat{H}_{n_{M a x}} \approx & \omega_{f} \hat{n} \otimes \mathbb{I}_{a}+\omega_{a} \mathbb{I}_{f} \otimes \hat{J}_{z}+\frac{\lambda}{\sqrt{N}}\left(\hat{a}+\hat{a}^{\dagger}\right) \otimes \hat{J}_{x}+\kappa\left(\hat{a}+\hat{a}^{\dagger}\right)^{2} \\
& \otimes \mathbb{I}_{a},
\end{aligned}
$$

where the matrix elements for the involved operators are

$$
\langle i|\hat{n}| j\rangle=j \delta_{i, j}, \quad\langle i|\hat{a}| j\rangle=\sqrt{j} \delta_{i, j-1}, \quad\left\langle i\left|\hat{a}^{\dagger}\right| j\right\rangle=\sqrt{j+1} \delta_{i, j+1},
$$

for $i, j=0,1, \ldots, n_{\operatorname{Max}}$ and

$$
\left\langle p\left|\hat{J}_{z}\right| q\right\rangle=q \delta_{p, q}, \quad\left\langle p\left|\hat{J}_{ \pm}\right| q\right\rangle=\sqrt{\frac{N}{2}\left(\frac{N}{2}+1\right)-m(m \pm 1)} \delta_{p, q \pm 1}
$$

with $\quad\left\langle p\left|\hat{J}_{x}\right| q\right\rangle=\frac{1}{2}\left\langle p\left|\hat{J}_{+}+\hat{J}_{-}\right| q\right\rangle \quad$ for $\quad p, q=-N / 2,-N / 2$ $+1, \ldots, N / 2-1, N / 2$. The symbols $\mathbb{I}_{f}$ and $\mathbb{I}_{a}$ stand for the unitary matrices of size $n_{M a x}+1$ and $N+1$ corresponding to the field and ensemble subspaces, in that order. The notation $\cdot \otimes \cdot$ is used for the tensor or Kronecker product.

As an eigenstate of the truncated version of the studied Hamiltonian, Eq. (21), can be easily verified to be, or not, an eigenstate for the exact full Hamiltonian, Eq. (3), the numerical approach taken here consists of assessing a maximum number of allowed excitations for the field, $n$, set to deliver at most a maximum error parameter, $\varepsilon$ $=\left|E_{1}-\langle\hat{H}\rangle_{j}\right| /\left|\langle\hat{H}\rangle_{j}\right|$, for a wide range of the phase space set by the coupling and nonlinear parameters, $(\lambda, \kappa)$ in that order. The set $\left\{\left(E_{j},\left|\psi_{j}\right\rangle\right)\right\}$ are the numerical eigenvalues and eigenstates, respectively, of the truncated Hamiltonian sorted in ascending order, $E_{j} \leqslant E_{j+1}$ for $j$ $=1, \ldots,(N+1)(n+1)$, and the notation $\langle\cdot\rangle_{j} \equiv\left\langle\psi_{j}|\cdot| \psi_{j}\right\rangle$ is used. In addition, a degeneracy parameter $\varepsilon_{d}=\mid E_{n}$ $-E_{1}|/| E_{1} \mid$ is established to discriminate between nondegenerate and degenerate ground states. In the latter case, the proper ground state is constructed as the normalized direct sum of the degenerate eigenstates.
In the following numerical analysis, the error and degeneracy parameters are set to the values $\varepsilon \leqslant 10^{-10}$ and $\varepsilon_{d} \leqslant 10^{-10}$. Numerical results for the on-resonance, $\omega_{f}$ $=\omega_{a}$, and off-resonance, $\omega_{f} \in[0.85,1) \omega_{a}$, case are performed for an assorted collection of parameters, $N$ $\in[2,6], \lambda / \sqrt{N} \in[0,5] \omega_{a}, \quad \kappa \in[0,5] \omega_{a}$. By fixing the coupling parameter at the largest value to be sampled and probing a few weak values of the nonlinear parameter, a maximum of two hundred excitations for the field, $n_{\operatorname{Max}}$ $=200$, is found to fulfill the required error and degeneracy parameters. Along the sampling of the phase space, both the error and degeneracy parameters are found to be in the required range. For the sake of brevity, only those results pertaining to a bipartite and a pentapartite ensemble are shown in Fig. 1 and in Fig. 2, respectively.

The mean value of the z-component of the angular momentum, $\left\langle\hat{J}_{z}\right\rangle$, which will be called population difference from now on, is shown in Fig. 1(a). Simulation results reveal that, as derived in the weak coupling regime, $\lambda \ll \omega_{a}$, the population difference is minimal, $\left\langle\hat{J}_{z}\right\rangle=-N / 2$, i.e., each and every two-level system is in its ground state and independent of the nonlinear parameter $\kappa$. Also, for a sufficiently large coupling, $\lambda \gg \omega_{a}$, along with an adequate nonlinear parameter $\kappa \ll\left(4 \lambda^{2}-\omega_{a} \omega_{f}\right) / 4 \omega_{a}$ such that $\lambda \gg \kappa$, the population difference is null, $\left\langle\hat{J}_{z}\right\rangle=0$, which relates to the ground state derived in the strong coupling regime, Eq. (19), under the aforementioned restrictions. Figure 1(b) shows that the numerical mean photon number for the field, $\langle\hat{n}\rangle$, is in agreement with the general behavior found in the analytical results; i.e., the field is in the vacuum field state, with a small two-photon component depending on the strength of the nonlinear parameter, for the weak coupling regime and in a coherent state, with mean photon number $|\alpha|^{2}$, for the strong coupling regime.

In order to demonstrate the existence of entanglement for the studied Hamiltonian in a moderate coupling regime, we calculate the maximum shared bipartite concurrence following the entangled web approach [41], Fig. 1(c), and the field-ensemble entanglement probed through von Neumann entropy of the reduced two-level ensemble, also known as entropy of entanglement [42], Fig. 1(d). Non-zero regions for both the bipartite concurrence and the entropy of entanglement are found between the separable states corresponding to the weak and strong coupling regimes, approximately delimited by the black curves in Figs. 1(c) and 1(d). It is possible to see that the maximum shared bipartite concurrence locates in the upper diagonal region, Fig. 1(c), indicating that the entanglement shared between the ensemble components occurs because of an approximately equal balance between the linear atom-photon and nonlinear photon-photon interactions. Instead, the entropy, which has its maximum value below the diagonal region, Fig. 1(d), shows a maximum entanglement between the two-level system ensemble and the photon field as the result of a larger atom-photon interaction strength.

A shortcoming of the numerical approach shows up at this point. The area of zero entropy below the entangled phase is inversely proportional to the value of the degeneracy parameter, $\varepsilon_{d}$, mentioned above. Also, the error parameter, $\varepsilon$, increases as the nonlinear parameter $\kappa$ goes to 
$\left\langle\hat{J}_{z}\right\rangle$

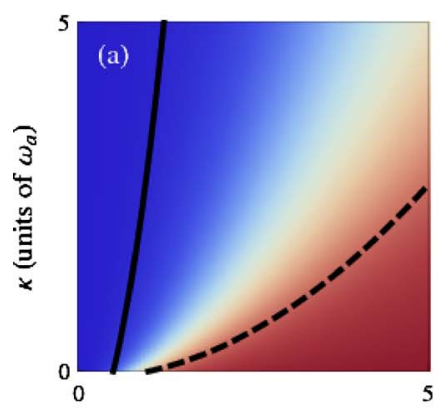

$\langle\hat{n}\rangle$

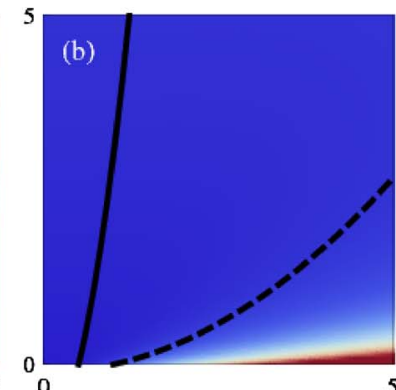

Concurrence

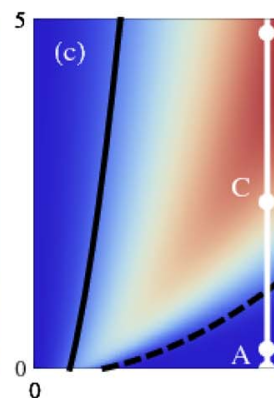

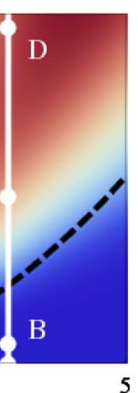

$\langle\hat{S}\rangle$

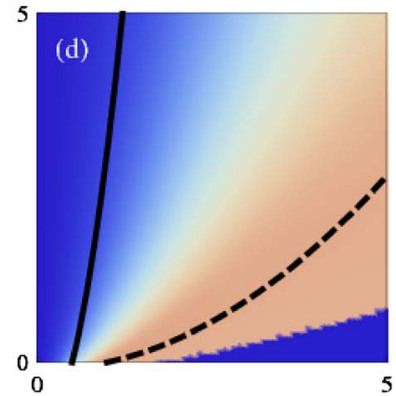

$\lambda N^{-1 / 2}$ (units of $\omega_{a}$ )
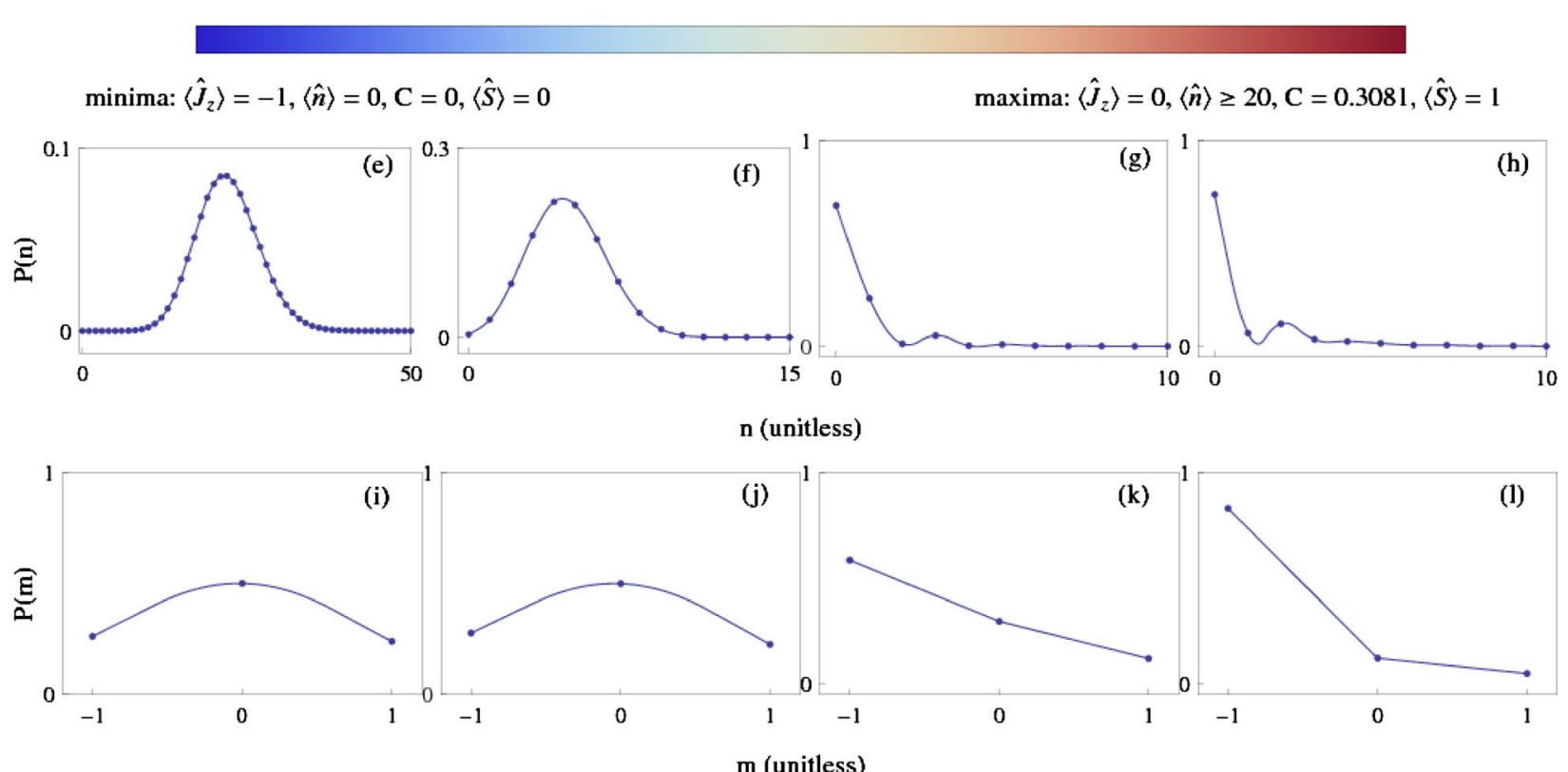

Fig. 1. (Color online) Phase diagram of our finite-size Dicke Hamiltonian in the parameter space of the linear photon-atom coupling strength, $\lambda$, and the nonlinear photon-photon interaction strength, $\kappa$. (a) Mean value for the atomic $z$-component, $\left\langle\hat{J}_{z}\right\rangle$. (b) Average photon number for the field, $\langle\hat{n}\rangle$. (c) Bipartite concurrence and (d) entropy of entanglement, $\langle\hat{S}\rangle$, are calculated for the case of $N=2$. The corresponding minima and maxima values for the color legend are shown below. The field photon number and atomic angular momentum probability distributions along the solid curve in (c) are shown in (e-h) and (i-l), ordered according to the markers $A-D$, respectively.

zero. These shortcomings appear because of the truncation of the Hilbert space for solving the eigenvalue problem. When the counter-rotating and diamagnetic like terms are neglected, the system is confined to certain finite subspaces and the numerical approach does not present this problem [16]. Numerical results might be improved by allowing a larger value for the maximum excitation of the field, optimizing the code, or effecting a customized analytical progressive diagonalization scheme based on those presented in $[43,44]$.

Nevertheless, the current approach allows the calculation of the states for the field and atomic ensemble up to the desired precision. In the second row of Fig. 1, we show the photon number probability distributions, $P(n)$ $=\left|\left\langle n \mid \psi_{g}\right\rangle\right|^{2}$, related to the four markers, labeled from $A$ to $D$, along a constant coupling parameter, $\lambda=3.323$, represented by the solid curve in Fig. 1(c). In the absence of nonlinear coupling, $\kappa=0$, a Poissonian photon number distribution is discovered in Fig. 1(e). By calculating the field quadrature variances $\left\langle\Delta \hat{X}^{2}\right\rangle$ and $\left\langle\Delta \hat{Y}^{2}\right\rangle$, with the field quadratures defined as $\hat{X}=\hat{a}^{\dagger}+\hat{a}$ and $\hat{Y}=\imath\left(\hat{a}^{\dagger}-\hat{a}\right)$, the field squeezing is probed. In this case, $\kappa=0$, the field is in a coherent state, as expected; i.e., the field quadratures' mean values and their uncertainty relation all have a value of one. With a small value of the nonlinear coupling strength, $\kappa=0.3$, the statistics for the photon number distribution becomes sub-Poissonian, shown in Fig. 1(f). The field is in a squeezed coherent state as the uncertainty relation for the field quadratures remains minimal, but the variance $\left\langle\Delta \hat{X}^{2}\right\rangle$ increases as $\left\langle\Delta \hat{Y}^{2}\right\rangle$ decreases. By increasing the nonlinear coupling, $\kappa=2.4$, an oscillating photon number distribution is found in Fig. $1(\mathrm{~g})$. Now, the quadrature squeezing seems to be reversed and the variance $\left\langle\Delta \hat{Y}^{2}\right\rangle$ is smaller than $\left\langle\Delta \hat{X}^{2}\right\rangle$ and close to a value of one. Also, as the value for the quadratures' uncertainty relation is more than one, the field is no longer in a coherent state. For further increase of the nonlinear coupling, $\kappa=4.8$, the oscillating photon number distribution remains, Fig. $1(\mathrm{~h})$, the variance $\left\langle\Delta \hat{Y}^{2}\right\rangle$ is further squeezed, 
$\left\langle\hat{J}_{2}\right\rangle$

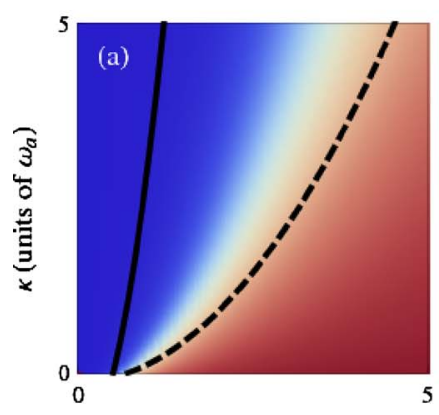

$\langle\hat{n}\rangle$

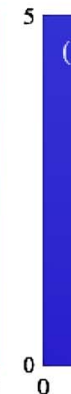

Concurrence

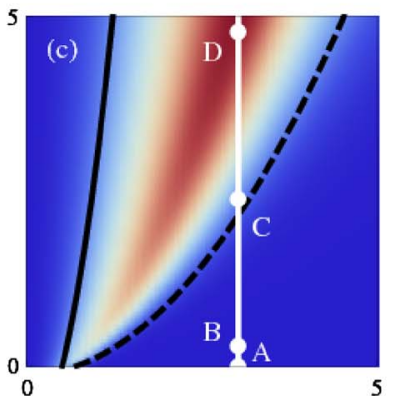

$\langle\hat{S}\rangle$

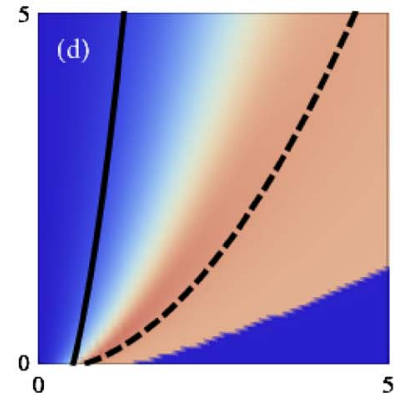

$\lambda N^{-1 / 2}$ (units of $\omega_{a}$ )

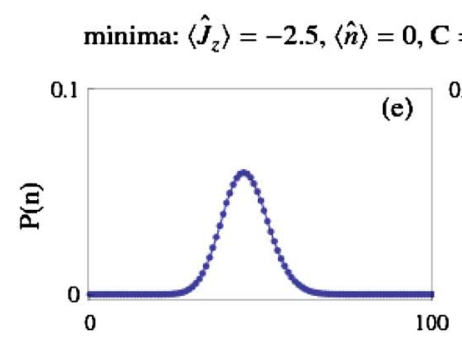

$=0,\langle\hat{S}\rangle=0$

$\operatorname{maxima}:\left\langle\hat{J}_{z}\right\rangle=0,\langle\hat{n}\rangle \geq 20, \mathrm{C}=0.1076,\langle\hat{S}\rangle=0.8204$

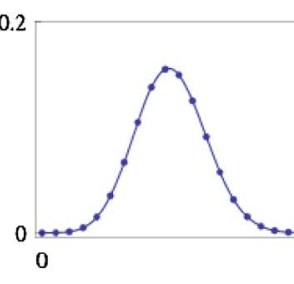

(f)

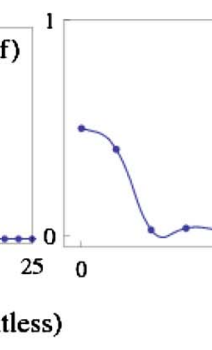

(g)

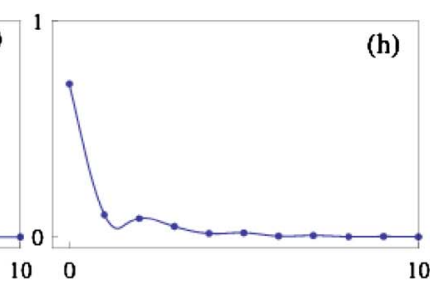

$\mathrm{p}$ (unitless)
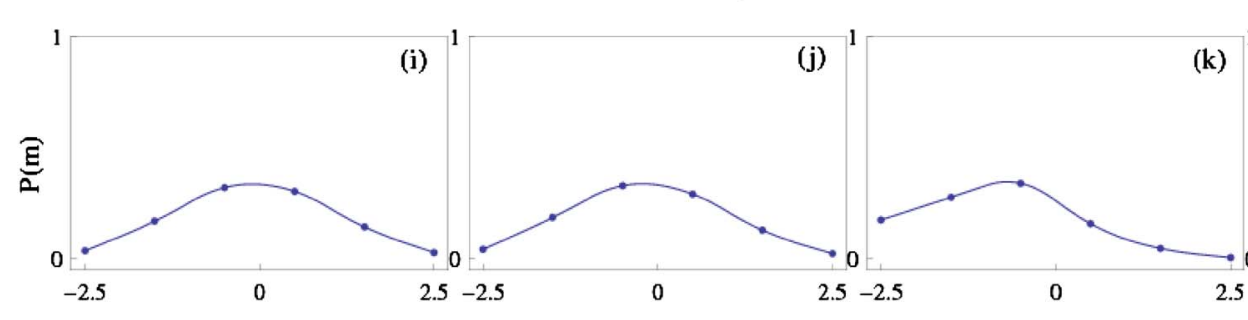

(k)

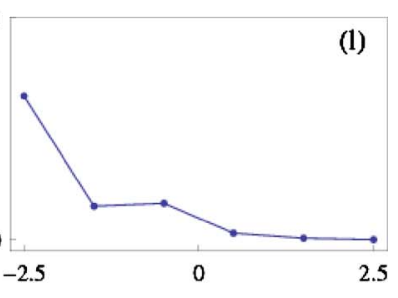

m (unitless)

Fig. 2. (Color online) Same as Fig. 1, but for the case of $N=5$.

and the field is not a coherent state but shows a tendency to become the superposition of the vacuum and twophoton state.

Besides the photon number probability distributions, the corresponding z-component angular momentum probability distributions, $P(m)=\left|\left\langle m \mid \psi_{g}\right\rangle\right|^{2}$, are shown in the third row of Fig. 1, in the same order related to the four points $A$ to $D$ along the solid curve in Fig. 1(c). We calculate the mean values and variances for the three momentum operators, $\left\langle\hat{J}_{i}\right\rangle$ and $\left\langle\Delta \hat{J}_{i}^{2}\right\rangle$ for $i=x, y, z$, as well as the uncertainty relation between the population difference and the dipole phase, $4\left\langle\Delta \hat{J}_{z}^{2}\right\rangle\left\langle\Delta \hat{\Phi}^{2}\right\rangle \geqslant 1$, where $\left\langle\Delta \hat{\Phi}^{2}\right\rangle$ $=\left\langle\Delta \hat{J}_{y}^{2}\right\rangle /\left\langle\hat{J}_{x}\right\rangle^{2}$. Again, by increasing the nonlinear coupling strength, Figs. 1(i)-1(1), the atomic state changes from a coherent atomic state in the absence of the nonlinear parameter to a squeezed coherent atomic state for a small nonlinear parameter. For a larger nonlinear coupling strength, the squeezed atomic state becomes a state where the minimal Dicke state, $|N,-N / 2\rangle$, predominates. Our simulation results indicate the co-existence of squeezed fields and squeezed atomic ensembles in the moderate coupling regime. The field and atomic statistics for the points discussed above, approximated to three decimal points for the sake of space, are shown in Table 1.
As the number of two-evel systems increase, e.g., $N=5$ in Fig. 2, the maximum bipartite entanglement shared between ensemble components seems to be inversely proportional to the ensemble size, and the region of entanglement decreases. In the second and third rows of Fig. 2, similar photon and atomic statistics, from Poissonian and sub-Poissonian to oscillating photon number distributions for the field, and from the coherent to squeezed atomic ensembles, respectively, are demonstrated along a constant coupling parameter, $\lambda=3.019$.

\section{CONCLUSION}

Two phase transitions for the ground state were found for a finite size Dicke Hamiltonian plus counter-rotating and quadratic field terms, corresponding to the weak and strong coupling regimes. The ground states before and after these transitions are analytically found to be pure separable states (thus there exists no entanglement in the system). The states are differentiated from each other by both the state of the field and two-level system ensemble; i.e., the superposition of the vacuum and two photon field states times all the components of the ensemble 
Table 1. Field and Atomic Statistics for the Markers $A$ to $D$ in Fig. 1(c) for $N=2$ and in Fig. 2(c) for $N=5$ Two-Level Systems ${ }^{a}$

\begin{tabular}{|c|c|c|c|c|c|c|c|c|}
\hline & \multicolumn{4}{|c|}{$\begin{array}{c}2 \text { TLS, Fig. 1(e)-1(1) } \\
\lambda=3.323\end{array}$} & \multicolumn{4}{|c|}{$\begin{array}{c}5 \text { TLS, Fig. 2(e)-2(l) } \\
\lambda=3.019\end{array}$} \\
\hline & $\underset{\kappa=0}{\mathrm{~A}}$ & $\begin{array}{c}\mathrm{B} \\
\kappa=0.3\end{array}$ & $\begin{array}{c}\mathrm{C} \\
\kappa=2.4\end{array}$ & $\underset{\kappa=4.8}{\mathrm{D}}$ & $\underset{\kappa=0}{\mathrm{~A}}$ & $\underset{\kappa=0.3}{\mathrm{~B}}$ & $\begin{array}{c}\mathrm{C} \\
\kappa=2.4\end{array}$ & $\underset{\kappa=4.8}{\mathrm{D}}$ \\
\hline$\langle\hat{n}\rangle$ & 22.078 & 4.590 & 0.502 & 0.678 & 45.528 & 9.417 & 0.731 & 0.707 \\
\hline$\left\langle\Delta \hat{n}^{2}\right\rangle$ & 22.084 & 3.155 & 1.011 & 2.135 & 45.545 & 6.416 & 1.153 & 2.162 \\
\hline$\left\langle\Delta \hat{X}^{2}\right\rangle$ & 1.000 & 0.676 & 1.824 & 2.941 & 1.000 & 0.676 & 1.645 & 2.609 \\
\hline$\left\langle\Delta \hat{Y}^{2}\right\rangle$ & 1.000 & 1.481 & 0.869 & 0.355 & 1.000 & 1.480 & 1.087 & 0.426 \\
\hline$\left\langle\Delta \hat{X}^{2}\right\rangle\left\langle\Delta \hat{Y}^{2}\right\rangle$ & 1.000 & 1.000 & 1.586 & 1.045 & 1.000 & 1.000 & 1.789 & 1.112 \\
\hline$\left\langle\hat{J}_{x}\right\rangle$ & -1.000 & 0.000 & 0.000 & 0.000 & -2.499 & 2.495 & 0.000 & 0.000 \\
\hline$\left\langle\Delta \hat{J}_{x}^{2}\right\rangle$ & 0.000 & -0.052 & 0.903 & 0.756 & 0.001 & 0.005 & 5.630 & 3.576 \\
\hline$\left\langle\hat{J}_{y}\right\rangle$ & 0.000 & 0.000 & 0.000 & 0.000 & 0.000 & 0.000 & 0.000 & 0.000 \\
\hline$\left\langle\Delta \hat{J}_{y}^{2}\right\rangle$ & 0.5000 & 0.500 & 0.392 & 0.364 & 1.250 & 1.250 & 1.147 & 0.719 \\
\hline$\left\langle\hat{J}_{z}\right\rangle$ & -0.023 & -0.512 & -0.466 & -0.781 & -0.068 & -0.153 & -0.861 & -1.902 \\
\hline$\left\langle\Delta \hat{J}_{z}^{2}\right\rangle$ & 0.500 & 0.5000 & 0.487 & 0.269 & 1.250 & 1.246 & 1.232 & 0.837 \\
\hline $4\left\langle\Delta \hat{J}_{z}^{2}\right\rangle\left\langle\Delta \hat{\Phi}^{2}\right\rangle$ & 1.000 & $1.151 \times 10^{47}$ & $7.515 \times 10^{54}$ & $5.887 \times 10^{51}$ & 1.000 & 1.000 & $7.180 \times 10^{38}$ & $1.682 \times 10^{41}$ \\
\hline
\end{tabular}

\footnotetext{
${ }^{a}$ See the text for more details.
}

in the ground state for couplings less than the weak critical coupling, and a non-vacuum coherent field state times all the components of the ensemble in a superposition of ground and excited states for couplings larger than the strong critical coupling.

Between these extremes, the ground state presents both ensemble-field entanglement and bipartite entanglement between the ensemble components. Results on ensemble bipartite entanglement behave as expected; the degree of maximum shared pairwise entanglement decreases as the number of entangled pairs in the two-level ensemble increases; i.e., for a sufficiently large ensemble, e.g., the infinitely large ensemble considered in the thermodynamic limit, the maximum shared bipartite entanglement will tend to zero and there will be no intermediate region between the weak and strong regimes. Thus, the phase space region for which the ground state of the system is entangled is directly related to the finite size of the system.

\section{ACKNOWLEDGMENTS}

This work was supported by the National Tsing-Hua University under contract No. 98N2309E1 and the National Science Council of Taiwan under the contract No. 982112-M-007-001-MY3.

\section{REFERENCES}

1. C. Cohen-Tannoudji, J. Dupont-Roc, and G. Grynberg, Photons and Atoms (Wiley-VCH, 2004).

2. L. Allen and J. H. Eberly, Optical Resonance and Two-Level Atoms (Dover, 1975).

3. R. H. Dicke, "Coherence in spontaneous radiation processes," Phys. Rev. 93, 99-110 (1954).

4. I. Bialynicki-Birula and K. Rzažewski, "No-go theorem concerning the superradiant phase transition in atomic systems," Phys. Rev. A 19, 301-303 (1979).
5. K. Rzažewski and K. Wodkiewicz, "Stability of matter interacting with photons," Phys. Rev. A 43, 593-594 (1991).

6. K. Rzažewski, K. Wódkiewicz, and W. Zakowicz, "Phase transitions, two-level atoms, and the $a^{2}$ term," Phys. Rev. Lett. 35, 432-434 (1975).

7. K. Gawedzki and K. Rzažewski, "No-go theorem for the superradiant phase transition without dipole approximation," Phys. Rev. A 23, 2134-2137 (1981).

8. W. R. Mallory, "Solution of a multiatom radiation model using the bargmann realization of the radiation field," Phys. Rev. 188, 1976-1987 (1969).

9. K. Hepp and E. Lieb, "On the superradiant phase transition for molecules in a quantized radiation field: the Dicke maser model," Ann. Phys. 76, 360-404 (1973).

10. Y. K. Wang and F. T. Hioe, "Phase transition in the Dicke model of superradiance," Phys. Rev. A 7, 831-836 (1973).

11. K. Hepp and E. H. Lieb, "Equilibrium statistical mechanics of matter interacting with the quantized radiation field," Phys. Rev. A 8, 2517-2525 (1973).

12. C. Emary and T. Brandes, "Quantum chaos triggered by precursors of a quantum phase transition: The Dicke model," Phys. Rev. Lett. 90, 044101 (2003).

13. C. Emary and T. Brandes, "Phase transitions in generalized spin-boson (Dicke) models," Phys. Rev. A 69, 053804 (2004).

14. N. Lambert, C. Emary, and T. Brandes, "Entanglement and the phase transition in single-mode superradiance," Phys. Rev. Lett. 92, 073602 (2004).

15. J. Vidal and S. Dusuel, "Finite-size scaling exponents in the Dicke model," Europhys. Lett. 74, 817-822 (2006).

16. V. Bužek, M. Orszag, and M. Roško, "Instability and entanglement of the ground state of the Dicke model," Phys. Rev. Lett. 94, 163601 (2005).

17. T. Brandes, J. Inoue, and A. Shimizu, "Oscillatory behavior of a superradiating system coupled to electron reservoirs," Phys. Rev. Lett. 80, 3952-3955 (1998).

18. T. Vorrath and T. Brandes, "Dicke effect in the tunnel current through two double quantum dots," Phys. Rev. B 68, 035309 (2003).

19. F. Dimer, B. Estienne, A. S. Parkins, and H. J. Carmichael, "Proposed realization of the Dicke-model quantum phase transition in an optical cavity qed system," Phys. Rev. A 75, 013804 (2007).

20. K. Härkönen, F. Plastina, and S. Maniscalco, "Dicke model and environment-induced entanglement in ion-cavity," Phys. Rev. A 80, 033841 (2009). 
21. A. Blais, R.-S. Huang, A. Wallraff, S. M. Girvin, and R. J. Schoelkopf, "Cavity quantum electrodynamics for superconducting electrical circuits: An architecture for quantum computation," Phys. Rev. A 69, 062320 (2004).

22. G. Chen, Z. Chen, and J. Liang, "Simulation of the superradiant quantum phase transition in the superconducting charge qubits inside a cavity," Phys. Rev. A 76, 055803 (2007).

23. K. Baumann, C. Guerlin, F. Brennecke, and T. Esslinger, "Dicke quantum phase transition with a superfluid gas in an optical cavity," Nature (London) 464, 1301-1307 (2010).

24. D. Nagy, G. Konya, G. Szirmai, and P. Domokos, "Dickemodel phase transition in the quantum motion of a BoseEinstein condensate in an optical cavity," Phys. Rev. Lett. 104, 130401 (2010).

25. F. Illuminati, "Light does matter," Nat. Phys. 2, 803-804 (2006).

26. A. D. Greentree, C. Tahan, J. H. Cole, and L. C. L. Hollenberg, "Quantum phase transitions of light," Nat. Phys. 2, 856-861 (2006).

27. S.-C. Lei and R.-K. Lee, "Quantum phase transitions of light in the Dicke-Bose-Hubbard model," Phys. Rev. A 77, 033827 (2008).

28. M. J. Hartmann, F. G. S. L. Brandão, and M. B. Plenio, "Effective spin systems in coupled microcavities," Phys. Rev. Lett. 99, 160501 (2007).

29. M. J. Hartmann, F. G. S. L. Brandão, and M. B. Plenio, "A polaritonic two-component Bose-Hubbard model," New J. Phys. 10, 033011 (2008).

30. D. Rossini and R. Fazio, "Mott-insulating and glassy phases of polaritons in 1d arrays of coupled cavities," Phys. Rev. Lett. 99, 186401 (2007).

31. D. G. Angelakis, M. F. Santos, and S. Bose, "Photonblockade-induced Mott transitions and xy spin models in coupled cavity arrays," Phys. Rev. A 76, 031805 (2007).
32. S.-C. Lei, T.-K. Ng, and R.-K. Lee, "Photonic analogue of Josephson effect in a dual-species optical-lattice cavity," Opt. Express 18, 14586 (2010).

33. M. O. Scully and M. S. Zubairy, Quantum Optics (Cambridge University Press, 1997).

34. Y. Yamamoto and A. İmamoğlu, Mesoscopic Quantum Optics (Wiley Interscience, 1999).

35. T. C. Jarrett, A. Olaya-Castro, and N. F. J. Centre, "Optical signatures of quantum phase transitions in a light-matter system," Europhys. Lett. 77, 34001 (2007).

36. J. M. Knight, Y. Aharonov, and G. T. C. Hsieh, "Are superradiant phase transitions possible?," Phys. Rev. A 17, 14541463 (1978).

37. C. F. Lee and N. F. Johnson, "First-order superradiant phase transitions in a multiqubit cavity system," Phys. Rev. Lett. 93, 083001 (2004).

38. J. Keeling, "Coulomb interactions, gauge invariance, and phase transitions of the Dicke model," J. Phys. C: Condens. Matter 19, 295213 (2007).

39. F. T. Hioe, "Phase transitions in some generalized Dicke models of superradiance," Phys. Rev. A 8, 1440-1445 (1973).

40. O. Tsyplyatyev and D. Loss, "Dicke model: entanglement as a finite size effect," J. Phys.: Conf. Ser. 193, 012134 (2009).

41. M. Koashi, V. Bužek, and N. Imoto, "Entangled webs: Tight bound for symmetric sharing of entanglement," Phys. Rev. A 62, 050302(R) (2000).

42. M. Nielsen and I. Chuang, Quantum Information and Computation (Cambridge University Press, 2000).

43. F. Pan, M.-X. Xie, X. Guan, L.-R. Dai, and J. P. Draayer, "New exact solutions of the standard pairing model for welldeformed nuclei," Phys. Rev. C 80, 044306 (2009).

44. F. Pan, X. Guan, Y. Wang, and J. P. Draayerb, "A progressive diagonalization scheme for the Rabi Hamiltonian," arXiv:1004.2801v1 (2010). 